\title{
FISSION DYNAMICS WITH MICROSCOPIC LEVEL DENSITIES
}

Ward, D.; Carlsson, B.; Døssing, Thomas; Moller, P.; Randrup, J.; Aberg, S.

Published in:

Acta Physica Polonica B, Proceedings Supplement

DOI:

10.5506/APhysPolBSupp.10.201

Publication date:

2017

Document version

Publisher's PDF, also known as Version of record

Document license:

CC BY

Citation for published version (APA):

Ward, D., Carlsson, B., Døssing, T., Moller, P., Randrup, J., \& Aberg, S. (2017). FISSION DYNAMICS WITH

MICROSCOPIC LEVEL DENSITIES. Acta Physica Polonica B, Proceedings Supplement, 10(1), $201-2019$.

https://doi.org/10.5506/APhysPolBSupp.10.201 


\title{
Fission dynamics with microscopic level densities
}

\author{
Jørgen Randrup ${ }^{1, \star}$, Daniel Ward ${ }^{2}$, Gillis Carlsson², Thomas Døssing ${ }^{3}$, Peter Möller ${ }^{4}$, \\ and Sven Åberg ${ }^{2}$ \\ ${ }^{1}$ Lawrence Berkeley National Laboratory, Berkeley, California 94720, USA \\ ${ }^{2}$ Mathematical Physics, Lund University, 22100 Lund, Sweden \\ ${ }^{3}$ Niels Bohr Institute, 2100 Copenhagen Ø, Denmark \\ ${ }^{4}$ Los Alamos National Laboratory, Los Alamos, New Mexico 87545, USA
}

\begin{abstract}
Working within the Langevin framework of nuclear shape dynamics, we study the dependence of the evolution on the degree of excitation. As the excitation energy of the fissioning system is increased, the pairing correlations and the shell effects diminish and the effective potential-energy surface becomes ever more liquid-drop like. This feature can be included in the treatment in a formally well-founded manner by using the local level densities as a basis for the shape evolution. This is particularly easy to understand and implement in the Metropolis treatment where the evolution is simulated by means of a random walk on the five-dimensional lattice of shapes for which the potential energy has been tabulated. Because the individual steps between two neighboring lattice sites are decided on the basis of the ratio of the statistical weights, what is needed is the ratio of the local level densities for those shapes, evaluated at the associated local excitation energies. For this purpose, we adapt a recently developed combinatorial method for calculating level densities which employs the same single-particle levels as those that were used for the calculation of the pairing and shell contributions to the macroscopicmicroscopic deformation-energy surface. For each nucleus under consideration, the level density (for a fixed total angular momentum) is calculated microscopically for each of the over five million shapes given in the three-quadratic-surface parametrization. This novel treatment, which introduces no new parameters, is illustrated for the fission fragment mass distributions for selected uranium and plutonium cases.
\end{abstract}

\section{Introduction}

Soon after the discovery of nuclear fission in 1938 [1], it was recognized that the process can be viewed qualitatively as an evolution of the nuclear shape from that of a single compound nucleus to two receding fragments $[2,3]$ and that Langevin transport theory provides an appropriate model framework [3, 4]. A number of Langevin treatments of fission have been successfully developed and applied for excitations high enough to render the dynamics macroscopic, see for example Refs. [5-7].

If the collective shape dynamics is idealized as being highly dissipative, then the Langevin equation reduces to the Smoluchowski equation in which the evolution depends on the balance between the driving force provided by the potential energy of deformation and the dissipative force resulting from the coupling of the collective degrees of freedom to the remaining system. In this limit, it

^e-mail: JRandrup@LBL.gov 
has proven possible to describe the Brownian shape motion as a Metropolis walk on the associated multi-dimensional deformation-energy surface [8]. This calculationally simple method has yielded remarkably good results for the fission-fragment mass distributions [9] and it has made it possible to predict fission-fragment mass distributions for poorly explored regions of the nuclear chart $[10,11]$.

\section{Microscopic shape-dependent level density}

In Ref. [12] a method was developed for microscopic calculations of level densities for deformed nuclei and it has been adapted to the fission process [13]. For a specified shape $\chi$, the single-particle levels for protons and neutrons needed for the combinatorial calculation of the level density are obtained by solving the Schrödinger equation in the associated folded-Yukawa potential. These are the same levels as those previously used in Ref. [11] to calculate the microscopic shell and pairing energies in the construction of the deformation energy surface, thus guaranteeing consistency of the treatment. The corresponding local many-body vacuum state $|0 ; \chi\rangle$ has $N$ neutrons and $Z$ protons filling the lowest single-particle states and the uncorrelated excited states consist of all multiple particle-hole excitations,

$$
|i ; \chi\rangle=\prod_{n \geq 1} a_{v_{n}^{(i)} \mu_{n}^{(i)}}|0 ; \chi\rangle,
$$

For each many-body state $|i ; \chi\rangle$, blocked BCS calculations for neutrons and protons separately provide the state-dependent pairing gaps, $\Delta_{i}^{\mathrm{n}}(\chi)$ and $\Delta_{i}^{\mathrm{p}}(\chi)$, respectively, and the energy of the correlated intrinsic many-body state, $E_{i}(\chi)=E_{i}^{\mathrm{n}}(\chi)+E_{i}^{\mathrm{p}}(\chi)$.

For each such intrinsic state, the angular momentum along the nuclear symmetry axis is denoted by $K$ and it is assume that it forms a rotational band head. The resulting total angular momenta $I$ may then take on the values $I=K,(K+1),(K+2), \ldots$ and the corresponding rotational energies of the band members are

$$
E_{i}^{\mathrm{rot}}(I ; \chi)=\frac{I(I+1)-K_{i}(\chi)^{2}}{2 \mathcal{J}_{\perp}\left(\chi, \Delta_{i}^{\mathrm{n}}(\chi), \Delta_{i}^{\mathrm{p}}(\chi)\right)} .
$$

The moment of inertia $\mathcal{J}_{\perp}$ is approximated by the moment of inertia of a rigid body with the shape $\chi$, modified by the calculated pairing gaps for the state [14].

In Ref. [12] it was found that the enhancements arisng from collective vibrational modes are unimportant and they are therefore ignored in the present fission applications. The total energy of a state is then given by

$$
E_{i}(I, \chi)=E_{i}^{\mathrm{n}}(\chi)+E_{i}^{\mathrm{p}}(\chi)+E_{i}^{\mathrm{rot}}(I ; \chi) .
$$

For each shape $\chi$, the resulting states are binned according to their energy $E_{i}$ and their total angular momentum $I$; the bin width was taken as $\Delta E=200 \mathrm{keV}$. The sensitivity of our results to the bin size has been tested and it was found that a doubling or tripling of $\Delta E$ produces negligible changes in the calculated mass distributions.

In Fig. 1, we show calculated level densities versus the backshifted excitation energy for three special shapes for which the shell and apiring energies have different magnitudes. At the second minimum, the gaps in the single-particle spectra for neutrons and protons provide the special stability of this shape region, quantitatively expressed by a considerable negative shell correction energy. On the other hand, the same gaps render it costly in energy to make particle-hole excitations, resulting in a slow increase of the level density when the nucleus is excited. The opposite situation is encountered at the symmetric barrier, where a high density of single-particle states around the Fermi energy results in a large positive shell correction energy, while at the same time delivering particle-hole states at a low cost in energy, providing a rapid increase of the level density with local excitation energy. 


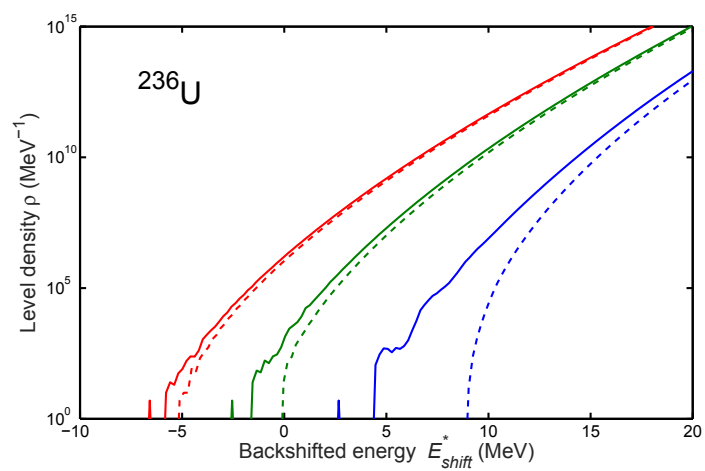

Figure 1. The microscopic level density, $\rho\left(E^{*}\right)$, calculated for ${ }^{236} \mathrm{U}$ at angular momentum $I=4 \hbar$, plotted versus the backshifted energy defined as $E_{\text {shift }}^{*}=E^{*}+E_{\mathrm{sh}}+E_{\mathrm{pc}}$ where $E_{\mathrm{sh}}$ is the shell energy and $E_{\mathrm{pc}}$ is the pairing correlation energy; three selected shapes are illustrated, either with (solid) or without (dashed) pairing: (i) the second minimum (red) $\left(E_{\mathrm{sh}}=-5.4 \mathrm{MeV}, E_{\mathrm{pc}}=-1.5 \mathrm{MeV}\right)$, (ii) the outer symmetric fission barrier (blue) $\left(E_{\mathrm{sh}}=\right.$ +8.7 MeV, $E_{\mathrm{pc}}=-6.1 \mathrm{MeV}$ ), and (iii) an intermediate shape (green) $\left(E_{\mathrm{sh}}=-0.3 \mathrm{MeV}, E_{\mathrm{pc}}=\right.$ $-2.5 \mathrm{MeV})$.

These opposite effects of the shell structure on the potential energy and on the level density cause the no-pairing results (dashed curves on Fig. 1) to converge towards the level density with zero shell energy, illustrating the suppression of shell energy with increasing exitation energy, as discussed in Refs. [15-17].

The effects on the level density from shell structure and pairing correlations subside steadily as the excitation energy is increased. Two different energy scales are involved, with the pairing disappearing at a lower excitation energy than the shell structure. The present study of fission shape evolution considers a large number $\left(>5 \cdot 10^{6}\right)$ of nuclear shapes and, consequently, a large variety of shell and pairing energies appear. It is therefore important to employ a level density that accounts for the changes in shell and pairing effects when the shape is varied. This can be ensured by employing a microscopic calculation of the level density up to sufficiently high excitation energies for all shapes considered. To reduce the required computation times, we employ an extrapolation procedure that takes account of the changes in pairing and shell effects with excitation energy for each shape.

Because the computational effort required by the combinatorial method grows exponentially with excitation, it is practically important to develop a simple way of extending the results to high energy. We do that by switching to an analytical expression at $E^{*}(\chi) \approx 6 \mathrm{MeV}$, below which most of the specific structure effects, such as spectral gaps or non-monotonic behavior, have disappeared. In this way, important structures in the level density are maintained, while the numerical calculations are kept to a manageable level (about 3 CPU seconds per shape). For each particular shape $\chi$, the extrapolation makes use of its specific shell and pairing energies, $E_{\mathrm{sh}}(\chi)$ and $E_{\mathrm{pc}}(\chi)$. Their influence diminishes with increasing energy and the level density approaches the analytical expression for a Fermi gas.

We employ the following simple analytical Fermi-gas expression [21], which is suitable for deformed nuclei with a fixed small angular momentum,

$$
\rho\left(N, Z, E^{*}(\chi), I\right)=C(\chi) \tilde{E}_{\text {intr }}^{-3 / 2} \exp \left(2 \sqrt{a_{0} \tilde{E}_{\text {intr }}}\right)
$$

where $E_{\text {intr }}=E^{*}(\chi)-I(I+1) \hbar^{2} / 2 \mathcal{J}_{\perp}(\chi)$ is the approximate intrinsic energy. Accounting for the different energy scales of shell effects and pairing effects, we introduce a backshifted intrinsic excitation energy $\tilde{E}_{\text {intr }}$, which is similar to the effective excitation $E_{\text {eff }}^{*}$ of Ref. [9] and can be considered as a generalization of the prescription originally employed by Ignatyuk et al. [17],

$$
\tilde{E}_{\text {intr }}(\chi)=E_{\text {intr }}(\chi)+\left(1-\mathrm{e}^{-E_{\text {intr }}(\chi) / E_{\mathrm{d}, \mathrm{sh}}}\right) E_{\mathrm{sh}}+\left(1-\mathrm{e}^{-E_{\text {intr }}(\chi) / E_{\mathrm{d}, \mathrm{pc}}}\right) E_{\mathrm{pc}} .
$$


Here $E_{\mathrm{sh}}(\chi)$ is the shell-correction energy and $E_{\mathrm{pc}}(\chi)$ is the pairing condensation energy, both calculated for the lowest state at the specified shape $\chi$. The full backshift, $\tilde{E}_{\mathrm{intr}}=E_{\mathrm{intr}}+E_{\mathrm{sh}}+E_{\mathrm{pc}}$ emerges when $E_{\text {intr }}$ is much larger than both damping scales.

The parameter $E_{\mathrm{d}, \mathrm{sh}}$ sets the energy scale for the melting of shell structure and the parameter $E_{\mathrm{d}, \mathrm{pc}}$ is the corresponding energy scale for the melting of pairing correlations. For simplicity, both quautities are assumed to be common to all shapes. By fitting the analytic expression for a number of typical shapes, optimal values of $E_{\mathrm{d}, \mathrm{sh}}$ and $E_{\mathrm{d}, \mathrm{pc}}$ were determined then applied for all shapes. The normalization constant $C(\chi)$ in Eq. (4) is determined by continuity with the corresponding microscopic value at the matching energy. The validity of the analytical extrapolation is illustrated in Fig. 2.

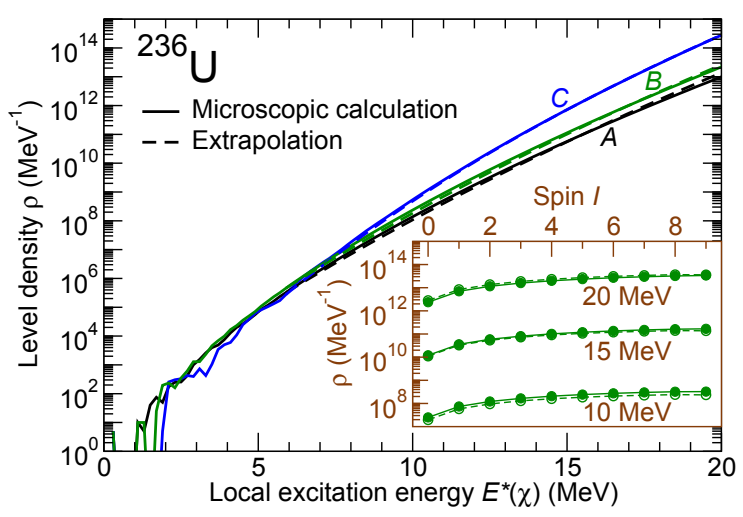

Figure 2. Microscopic level densities (solid lines) compared to extrapolated values (dashed lines). Three different deformations for ${ }^{236} U$ are considered: the second minimum $(A)$, the asymmetric second saddle $(B)$, and an elongated symmetric shape close to the outer barrier $(C)$. Extrapolated values are shown for local excitation energies $E^{*}(\chi) \geq 6 \mathrm{MeV}$. The inset shows the angular momentum dependence of the level density at the asymmetric saddle $(B)$ for different excitation energies.

\section{Brownian shape evolution}

The description of nuclear fission as a generalized Brownian motion builds on the assumption that the evolving nucleus can be characterized by its shape degrees of freedom $\chi$. The associated shape parameters are treated as classical variables that are coupled dissipatively to the remaining nuclear degrees of freedom which have a primarily microscopic character and are assumed to have a short relaxation time. The resulting large-amplitude collective motion then exhibits a strongly damped diffusive evolution that can be described as a random walk on the associated multi-dimensional potential-energy surface $U(\chi)$. When the values of the potential are available on a discrete lattice of shapes (as is the case for the 5D tabulations in Ref. [18]), the Smoluchowski equation can be approximately simulated by means of a random walk on this lattice [8].

Because the random walk must satisfy detailed balance, the following relation must hold between the rates of transition between one lattice site $\chi$ and another $\chi^{\prime}, v\left(\chi \rightarrow \chi^{\prime}\right)$, and the corresponding statistical weights which are proportional to the local level densities, $\rho(\chi)$,

$$
v\left(\chi \rightarrow \chi^{\prime}\right) / v\left(\chi^{\prime} \rightarrow \chi\right)=\rho\left(\chi^{\prime}\right) / \rho(\chi) .
$$

This condition can be satisfied in many ways and the Metropolis procedure [19] is merely one particularly simple possibility: A proposed shape change from $\chi$ to $\chi^{\prime}$ is accepted unconditionally if 
$\rho\left(\chi^{\prime}\right)>\rho(\chi)$ (corresponding to a "downhill" step), whereas it it accepted only with the probabilty $\rho\left(\chi^{\prime}\right) / \rho(\chi)$ otherwise (i.e. if the proposed step is "uphill"); it is readily verified that this procedure satisfies detailed balance.

In Ref. [8] and all subsequent applications until recently [13], simplified Fermi-gas level-density expressions were used, $\rho_{E}(\chi) \approx \exp [2 \sqrt{E-U(\chi)}]$. Because there is no explicit dependence on the shape, the ratio between neighboring level densities can be approximated by means of a logarithmic expansion,

$$
\frac{\rho\left(\chi^{\prime}\right)}{\rho(\chi)} \approx \exp \left[-\frac{\partial \ln \rho\left(E^{*}\right)}{\partial E^{*}} \frac{\partial U(\chi)}{\partial \chi} \Delta \chi\right] \approx \mathrm{e}^{-\Delta U / T},
$$

where $\Delta U=U\left(\chi^{\prime}\right)-U(\chi)$ is the change in the potential energy associated with the proposed shape change $\chi \rightarrow \chi^{\prime}=\chi+\Delta \chi$ and $T(\chi)=1 /\left[\partial \ln \rho\left(E^{*}\right) / \partial E^{*}\right]$ is the local nuclear temperature. In the new treatment [13], we wish to account for pairing correlations and shell effects and we therefore employ the microscopic level densities (see Sect. 2) to evaluate the required ratios $\rho\left(\chi^{\prime}\right) / \rho(\chi)$.

To obtain a general understanding of the Brownian shape evolution towards fission, we have studied trajectories for the case ${ }^{236} \mathrm{U}$ at $E^{*}=6.55 \mathrm{MeV}$. The aggregate number of visits to different sites on the shape lattice is shown in Fig. 3, projected onto the plane of mass asymmetry $\alpha_{\mathrm{g}}$ and elongation $q_{2}$. The solid black curve shows the mean "scission" elongation, i.e. the average elongation, $q_{2}$, for random walks terminating with a particular mass asymmetry $\alpha_{\mathrm{g}}$.

The potential-energy landscape plays a key role in the shape evolution. The Metropolis walks all start in the second potential-energy minimum at $\alpha_{\mathrm{g}}=0$ and $q_{2}=2.36$. From this starting point, they exhibit a diffusive track that probes the potential landscape at ever larger deformations, including the

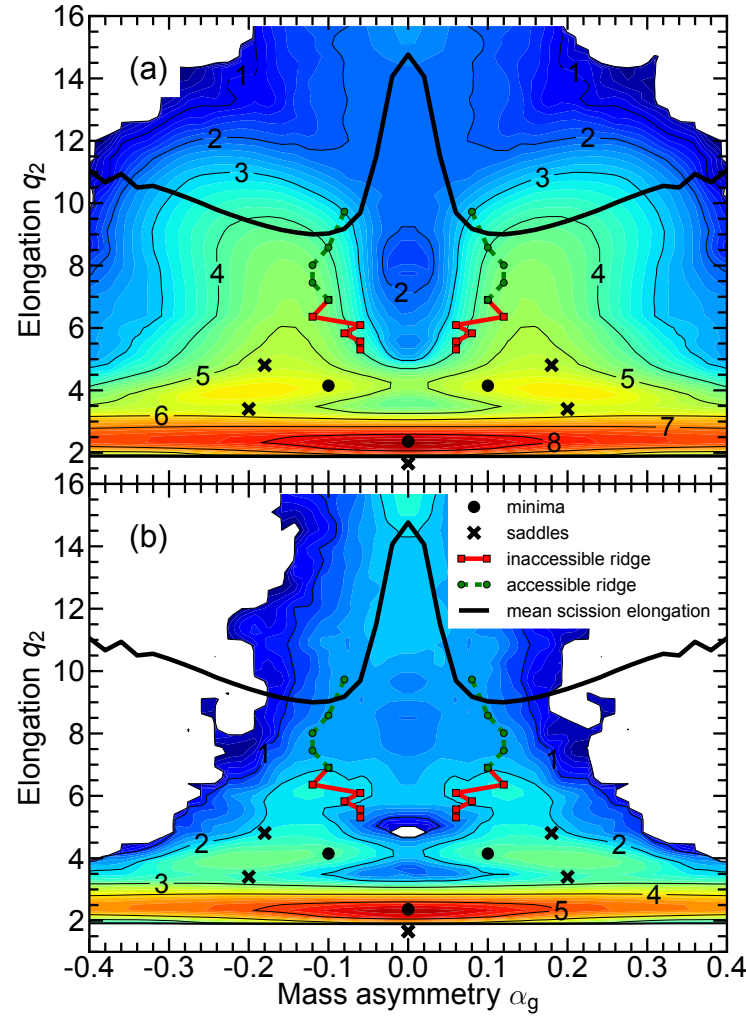

Figure 3. Contour diagrams (on a logarithmic scale) of the cumulative number of visits to shape lattice sites with asymmetry $\alpha_{\mathrm{g}}$ and elongation $q_{2}$ for ${ }^{236} \mathrm{U}$ at $E^{*}=S_{\mathrm{n}}=6.55 \mathrm{MeV}$. Panel (a) includes all tracks, whereas panel (b) contains only tracks that end in symmetric fission $\left(\alpha_{\mathrm{g}}=0\right)$. Energy minima are shown as solid circles, while saddle points are shown as crosses. The solid black curve shows the mean "scission" elongation (where the random walk is halted and the mass split binned). The ridge separating the symmetric valley from the asymmetric valleys is shown as a solid red/dashed green curve, where green/red indicates that the ridge is below/above the total energy $E^{*}$ and thus is either accessible or inaccessible, respectively. Symmetric fission events generally occur at larger elongations. This bimodal character of the mass distribution reflects the asymmetry dependence of the shellcorrection energy in the scission region: Wheeras for asymmetric splits the combined shell correction of the two prefragments encourages early neck formation, the more symmetric scission shapes tend to have a smaller shell energy. 
third minimum of potential energy located at an asymmetric shape having $\alpha_{\mathrm{g}} \approx \pm 0.1$ and $q_{2} \approx 4$. The distribution of site visits exhibits maxima in the regions around the second and third minima.

Beyond the outmost saddle point(s), around $\alpha_{\mathrm{g}} \sim \pm 0.2$ and $q_{2} \sim 5$, the potential starts to slope steadily down towards scission. Most often, a decreasing potential is accompanied by an increase in the level density and the Metropolis walk then changes its character from being diffusive in character to being drift dominated. As a result, "backwards" steps become unlikely and the average number of site visits tend to become relatively constant with elongation.

Concurrent with this steady drift in elongation, sideways diffusive steps are taken, thus providing some possibility for probing the potential with regard to changes in the fragment deformations, the neck radius, and, most important, the mass asymmetry $\alpha_{\mathrm{g}}$. A prominent region of shapes where this kind of evolution occurs is the interval $6<q_{2}<9,0.1<\alpha_{g}<0.24$ (displaying a rather uniform light green color on Fig. 3a).

Turning now to symmetric shapes, $\alpha_{\mathrm{g}} \approx 0$, we note that for the considered energy, $E^{*}=6.55 \mathrm{MeV}$, it is not possible to pass directly through the barrier region while keeping $\alpha_{\mathrm{g}}$ close to zero. Therefore, in order to reach elongated symmetric shapes, the nucleus must first acquire some degree of asymmetry. A comparison between the upper and lower parts of Fig. 3 shows that the diffusion process indeed proceeds in identical ways up to elongations of $q_{2} \approx 5$. But for elongations lager than $q_{2} \approx 5$, the potential landscape displays sloping asymmetric and symmetric valleys separated by a (sloping) ridge. The ridge location in the $\left(q_{2}, \alpha_{\mathrm{g}}\right)$ plane is indicated in Fig. 3, with the color of the ridge line indicating whether the ridge lies below (green) or above (red) the total energy. This implies that the distribution of tracks leading to symmetry is markedly different from the overall distribution.

\section{Energy dependence of the fragment yields}

The Metropolis walks were performed on a discrete lattice of more than five million shapes given in the three-quadratic-surface parametrization [20]. The five independent parameters characterizing a shape are the overall elongation of the nucleus (in terms of the reduced quadrupole moment $q_{2}$ ), the constriction of the central part (in terms of the neck radius $c$ ), the spheroidal deformations $\varepsilon_{\mathrm{f} 1}$ and $\varepsilon_{\mathrm{f} 2}$ of the two nascent fragments, and the reflection (mass) asymmetry $\alpha_{\mathrm{g}}$.

The use of microscopic level densities is particularly well-suited for studying the dependence of the shape evolution on the total excitation energy of the fissioning system because the microscopic pairing and shell effects automatically disappear the energy is raised.

The preliminary treatment in Ref. [9] used an energy-dependent effective potential,

$$
U_{E}(\chi)=U_{\text {macro }}(\chi)+\mathcal{S}\left(E^{*}(\chi)\right) E_{\text {micro }}(\chi),
$$

which is obtained by reducing the microscopic term with a suppression factor of the form $\mathcal{S}\left(E^{*}\right)=$ $(1+c) /\left[\exp \left(E^{*} / E_{\text {damp }}\right)+c\right]$, which decreases from one to zero as the local excitation $E^{*}=E-U(\chi)$ increases. The damping energy in the suppression fucntion was fixed to be $E_{\mathrm{damp}}=15 \mathrm{MeV}$ and the constant $c=20 \mathrm{MeV}$ was adjusted to ensure reproduction of the experimental yields for fission of ${ }^{234} U$ at $E^{*}=11 \mathrm{MeV}$ [23]. The local statistical weights are then proportional to a simplified Fermigas level density, $\rho_{E}(\chi) \sim \exp \left[2 \sqrt{E-U_{E}(\chi)}\right]$, and the Metropolis walk can thus be carried out as before without any other modifications of the code which is obviously very convenient. Although this approach yields quite reasonable results [9], it is unsatisfactory from a theoretical point of view because it fails to take account of the different energy scales of the pairing and shell effect as well as of their dependence on the nuclear shape.

Figure 4 shows the calculated charge yields for ${ }^{234} U$, as obtained by either using the simplified Fermi-gas level density based on the effective potential Eq. (8) introduced in Ref. [9] or the microscopic level densities [13]. The experimental data for ${ }^{233} U\left(n_{\text {th }}, f\right)[22]$ and ${ }^{234} U(\gamma, f)[23]$ are also 


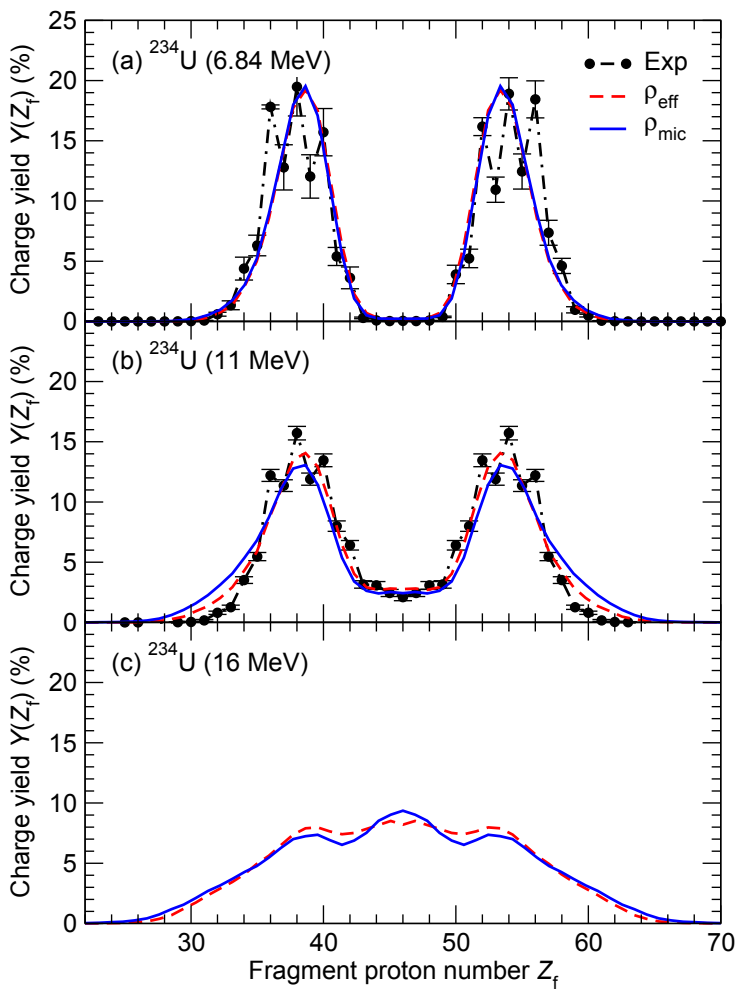

Figure 4. Fission-fragment charge distributions for ${ }^{234} \mathrm{U}$ at three different excitation energies $E^{*}(\mathrm{MeV}): 6.84$ (a), 11 (b), and 16 (c). The blue solid curves have been obtained with the microscopic level densities, while the dashed red curves were calculated with the effective level density $\rho_{\text {eff }}$ introduced in Ref. [9]. The results for $E^{*}=6.84 \mathrm{MeV}$ are compared to $\left(\mathrm{n}_{\mathrm{th}}, \mathrm{f}\right)$ data [22], while those for $E^{*}=11 \mathrm{MeV}$ are compared to $(\gamma, \mathrm{f})$ data [23]. The calculated results for $E^{*}=6.84 \mathrm{MeV}$ are practically identical and both are in good agreement with the experimental data. For $E^{*}=11 \mathrm{MeV}$ the present approach reproduces the symmetric yield of around $2 \%$, while the very asymmetric yields are too large. The calculated yields for $E^{*}=16 \mathrm{MeV}$ are also quite similar.

shown. The (n,f) results are similar (and in good agreement with the data). For $E^{*}=11 \mathrm{MeV}$, the current approach correctly reproduces the symmetric yield of around $2 \%$, whereas the yields for highly asymmetric splits are too large. The yields for $E^{*}=16 \mathrm{MeV}$ come out quite similar in both approaches.

The transition from asymmetric to symmetric fission with increasing energy in the actinides is usually associated with the "melting" of shell structure, with the yield gradually assuming the symmetric form characteristic of the macroscopic limit. This feature appears automatically when the microscopic level densities are used [13], as illustrated in Fig. 5 for fission of ${ }^{236} \mathrm{U}$ at various excitations. As the energy is increased from 6.55 to $11.55 \mathrm{MeV}$ there is a marked increase in the symmetric component because the shape diffuses ever more readily into the symmetric valley as the microscopic effects attenuate. At still higher energies the pairing and shell effects have largely subsided and the level density is determined by the excitation above the macroscopic potential, favoring symmetry. 


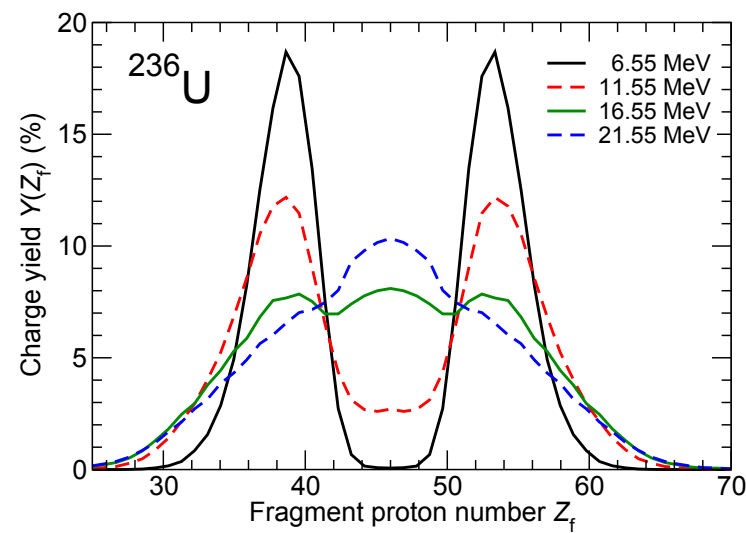

Figure 5. Calculated charge yields for fission of the nucleus ${ }^{236} \mathrm{U}$ at four values of the excitation energy extending from the neutron separation energy up to $E^{*} \approx 22 \mathrm{MeV}$. The calculated yields correspond to first-chance fission. At excitation energies above $\approx 11 \mathrm{MeV}$ the system is above the second-chance fission threshold, and it has an increasing probability of multi-chance fission as the energy is further increased. Consequently, the displayed results cannot be compared directly to experimental yields obtained with higher-energy neutrons impinging on ${ }^{235} \mathrm{U}$.

The importance of taking account of nuclear structure effects in the level density is well illustrated by the energy dependence of the fission-fragment yield for symmetric splits. Figure 6 displays results for the nucleus ${ }^{236} \mathrm{U}$ obtained with different strengths of the pairing interaction entering into the microscopic level densities. The standard calculation leads to a local maximum at $E^{*}=7 \mathrm{MeV}$, followed by a local minimum at $8 \mathrm{MeV}$; this bump is followed by a second bump ending with an inflection at around $10 \mathrm{MeV}$. The experimental data exhibit a qualitatively similar behavior, in particular a local maximum at $E^{*}=7 \mathrm{MeV}$. Without pairing, the absence of a gap produces a larger level density, especially for shapes with positive shell corrections, where both the gap and the single-particle level density are larger. The non-monotonic behavior of the symmetric yield has its origin in the structure of the microscopic level density related to pairing. It is thus evident that the symmetric yield is highly sensitive to the pairing properties of the level density.

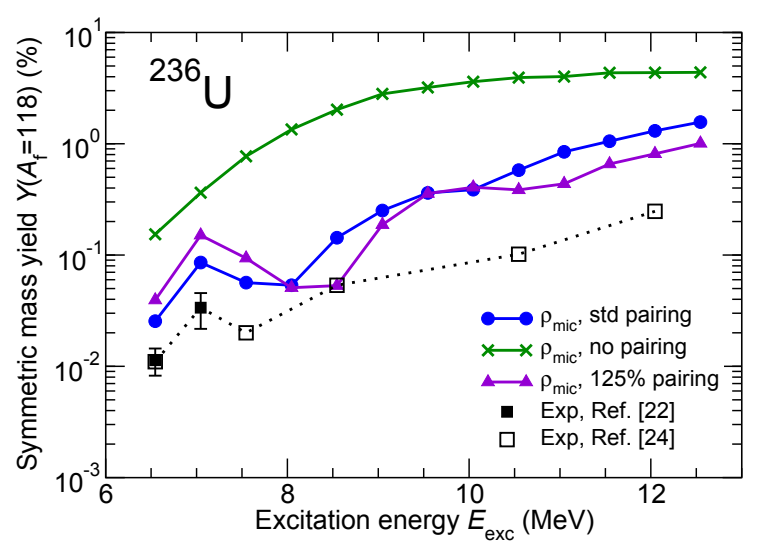

Figure 6. The dependence of the symmetric mass yield for ${ }^{236} \mathrm{U}$ on the excitation energy. In addition to the standard calculation (filled blue circles) are shown two additional calculations: one where the level densities are obtained without pairing (green crosses) and one where the pairing strength is $25 \%$ above the standard value (purple triangles). (It is the same potential-energy surface that has been used in all three calculations, namely the surface obtained with the standard pairing strength [18].) Also shown are the experimental data from Refs. [22] (solid squares) and [24] (open squares).

\section{Conclusions and outlook}

We have refined the Metropolis-walk approximation to the Brownian-motion treatment of fission dynamics [8] by employing microscopic local level densities obtained by the recently developed combinatorial method [12]. Because the single-particle levels used are the same as those employed for the macroscopic-microscopic calculation of the potential-energy surfaces, the approach is consistent and 
no new parameters need be introduced. Moreover, the combinatorial procedure provides access to the level density for a fixed value of angular momentum.

In order to reduce the considerable computing effort required for calculating the level density up to sufficiently high excitations, we employ an analytical extension above $\approx 6 \mathrm{MeV}$ where the combinatorial level density has become smooth. This procedure uses a damped backshift of the excitation energy based on the local pairing and shell energies. In this manner, we have obtained the local level density $\rho(N, Z, E, I ; \boldsymbol{\chi})$ for over 5 million shapes of several nuclei in the uranium region and for angular momenta up to $I=9$.

Using these parameter-free microscopic level densities with the Metropolis-walk method, we calculate fission fragment charge distributions for ${ }^{226} \mathrm{Th},{ }^{234,236} \mathrm{U}$, and ${ }^{240} \mathrm{Pu}$. The agreement with experimental data was on par with or better than what was obtained previously with a phenomenological level-density parametrization [9]. The angular-momentum dependence of the fission yields was found to be relatively small and decreasing with increasing excitation energy.

Because the microscopic level densities automatically contain the diminishing effects of pairing correlations and shell structure, the present refined model makes it possible to make more detailed predictions for the energy dependence of the fission yields. The gradual transition from asymmetric to symmetric fission and the detailed energy dependence of the symmetric yield were studied.

Particularly interesting is the finding that the symmetric fragment yield is not monotonically increasing with excitation energy. This perhaps counter intuitive effect appears to be a result of the large pairing correlations for shapes with positive shell-correction energies separating the asymmetricfission path from symmetric shapes. It is in this connection intriguing that a recent experiment has reported a non-monotonic energy dependence of the asymmetric peak shape in the fragment mass yields for fission of ${ }^{240} \mathrm{Pu}$ [25]. We plan to investigate these phenomena in more detail.

The present refined model provides a consistent and computationally manageable theoretical framework for studying large-scale collective motion of warm nuclear systems far from equilibrium and, in particular, it provides a unique tool for calculating energy-dependent fission fragment mass distributions. The present work extends the use of microscopic level densities from nuclei in shape equilibrium to arbitrary shapes and our present studies have revealed the intriguing possibility that the pairing interaction in shapes far from equilibrium. may manifest itself in a measurable manner through the energy dependence of the fisssion yields.

We are grateful to N. Schunck, A. Tonchev, and R. Vogt for discussions, helpful comments, and valuable suggestions. This work was supported by the Swedish Natural Science Research Council (BGC and SÅ), by the National Nuclear Security Administration of the U.S. Department of Energy at Los Alamos National Laboratory under Contract No. DE-AC52-06NA25396 (PM), and by the Office of Nuclear Physics in the U.S. Department of Energy(tm)s Office of Science under Contract No. DE-AC02-05CH11231 (JR).

\section{References}

[1] O. Hahn and F. Straßmann, Naturwiss. 27, 11 (1939)

[2] L. Meitner and O.R. Frisch, Nature 143, 239 (1939)

[3] N. Bohr and J.A. Wheeler, Phys. Rev. 56, 426 (1939)

[4] H.A. Kramers, Physica VII, 284 (1940)

[5] A.V. Karpov et al., Phys. Rev. C 63, 054610 (2001)

[6] P.N. Nadtochy et al., Phys. Rev. C 65, 064615 (2002)

[7] E.G. Ryabov et al., Phys. Rev. C 78, 044614 (2008)

[8] J. Randrup and P. Möller, Phys. Rev. Lett. 106, 132503 (2011) 
[9] J. Randrup and P. Möller, Phys. Rev. C 88, 064606 (2013)

[10] P. Möller, J. Randrup, and A.J. Sierk, Phys. Rev. C 85, 024306 (2012)

[11] P. Möller and J. Randrup, Phys. Rev. C 91, 044316 (2015)

[12] H. Uhrenholt, S. Åberg, A. Dobrowolski, Th. Døssing, T. Ichikawa, and P. Möller, Nucl. Phys. A 913, 127 (2013)

[13] D. Ward, G. Carlsson, Th. Døssing, P. Möller, J. Randrup, and S. Åberg, Phys. Rev. C 95, 024618 (2017)

[14] R. Bengtsson and S. Åberg, Phys. Lett. B 172, 277 (1986)

[15] J.R. Huizenga and L.G. Moretto, Ann. Rev. Nucl. Sci. 22, 427 (1972)

[16] A. Bohr and B.R. Mottelson, Nuclear Structure II, Benjamin, Reading, MA (1974)

[17] A.V. Ignatyuk, K.K. Istekov, and G.N. Smirenkin, Sov. J. Nucl. Phys. 29, 450 (1979)

[18] P. Möller, A. J. Sierk, T. Ichikawa, A. Iwamoto, R. Bengtsson, H. Uhrenholt, and S. Åberg, Phys. Rev. C 79, 064304 (2009)

[19] N. Metropolis, A.W. Rosenbluth, M.N. Rosenbluth, A.H. Teller, and E. Teller, J. Chem. Phys. 21, 1087 (1953)

[20] J.R. Nix, Nucl. Phys. A 130, 241 (1969)

[21] T. Ericsson, Adv. Phys. 9, 425 (1960)

[22] M.B. Chadwick et al. Nucl. Data Sheets, 112, 2887 (2011)

[23] K.-H. Schmidt et al., Nucl. Phys. A 665, 221 (2000)

[24] L.E. Glendenin, J.E. Gindler, D.J. Henderson and J.W. Meadows, Phys. Rev. C 24, 2600 (1981)

[25] M.E. Gooden et al., Nucl. Data Sheets 131, 319 (2016) 